\title{
ARTIFICIAL INTELLIGENCE AND GEOSPATIAL ANALYSIS IN DISASTER MANAGEMENT
}

\author{
M. Ivić ${ }^{1}$ \\ ${ }^{1}$ Faculty of Civil Engineering, Architecture and Geodesy, University of Split, Matice hrvatske 15, 21000 Split, Croatia - \\ majda.ivic@gradst.hr
}

KEY WORDS: Artificial intelligence, machine learning, geospatial analysis, disaster management, remote sensing

\begin{abstract}
:
For quick and efficient response, as well as for recovery after any natural or artificial catastrophe, one of the most important things are accurate and reliable spatial data in real or near real-time. It is essential to know the location as well as to track and analyse passive and active threats to quickly identify the possible dangers and hazards. As technology evolves and advances, there is a broader spectrum of sensors that provide spatial data, and nowadays, decision-making processes also include nontraditional, informal sources of information. Apart from the offer, demand for new spatial data is increasing as well. For quicker and enhanced integration and analysis of data, artificial intelligence (AI) tools are increasingly used which, in addition to immediate rapid reactions, can help to make better and smarter decisions in the future. Such software algorithms that imitate human intelligence can help in generating conclusions from natural phenomena presented by spatial data. Using AI in the data analysis can identify risk areas and determine future needs. This paper presents an overview of the use of AI in geospatial analysis in disaster management.
\end{abstract}

\section{INTRODUCTION}

Natural disasters are not so rare events and happen all around the world causing a lot of material damage and, unfortunately, sometimes loss of human lives. In dealing with such unwanted events, the most important thing is existence of different kinds of data, which are nowadays available more than ever before. With the development of technology and sensors, today data are obtained from many different sources. In order to respond quickly to a disaster, and to make adequate evacuation, and later on recovery plans, accurate and up-to-date spatial data are required. It is also important to know the location, as well as to track and analyse passive and active threats in order detect and identify the possible dangers and hazards in time.

Sometimes it is necessary to combine and integrate data from different data sources. For example, it may be needed to analyse coupled satellite imagery, drone imagery and seismic sensor data. Moreover, in disaster management, data analysis is often needed in real-time or near real-time, which is not an easy task when performed manually. Such and similar issues can be addressed by applying methods of artificial intelligence. Artificial intelligence algorithms, that imitate human intelligence, have become very popular among researches dealing with different tasks in disaster management.

The first part of the paper defines and describes the concept of artificial intelligence, as well as its types and most important subfields. The second part of the paper gives an overview of the use of AI in geospatial analysis in disaster management through the overview of the case studies.

\section{ARTIFICIAL INTELLIGENCE}

In 1955, computer scientist John McCarthy (Dartmouth College), "the father of artificial intelligence", in partnership with Marvin Minsky (Harvard University), Nathaniel Rochester (IBM), and Claude Shannon (Bell Telephone Laboratories), wrote "A proposal for the Dartmouth summer research project on artificial intelligence" (McCarthy et al. 1955). The Dartmouth Conference, at which the term "Artificial Intelligence" (AI) was first adopted, was held a year later (Ray, 2018). Nowadays, with the advancement of technology, availability of computers and increasing number of sensors and observed data, AI gets more and more attention.

According to McCarthy (2007) AI is "the science and engineering of making intelligent machines, especially intelligent computer programs." In a broadest sense, AI is a way of making a machine or artifact perform functions that are similar to human thoughts (Mellit, Kalogirou, 2007).

AI can be classified in many different ways but there are two types of classification that are mainly used. The first one, which is based on capabilities, categorizes AI in three groups: Weak AI or Narrow AI, General AI and Super AI. The most common and currently available AI is Narrow AI, which is a type of AI that can perform only one task for which it was trained. General $\mathrm{AI}$ is a type of AI that can perform any intellectual task and function as good as a human being, while Super AI can even surpass human intelligence. Researchers are now trying to develop machines with General AI, and Super AI is still a hypothetical concept (JavaTpoint, 2019).

Type two classification is based on functionality and divides AI into four categories: Reactive Machines, Limited Memory, Theory of Mind and Self-Awareness. Reactive Machines are the oldest type of AI systems that do not have memory-based functionality and are not capable of "learning". They can only automatically respond to a set of inputs. Almost all present-day AI applications belong to the category of Limited memory machines, which can learn from the past data in order to make decisions. For now, Theory of mind and Self-Aware machines are just a concepts. Theory of mind AI will be able to achieve better interaction by distinguishing needs, emotions, beliefs, and thought processes of the other side. Self-aware machines will be super intelligent and have self-awareness, i.e. it will have its own emotions, needs, beliefs, and potentially desires (Joshi, 2019). 


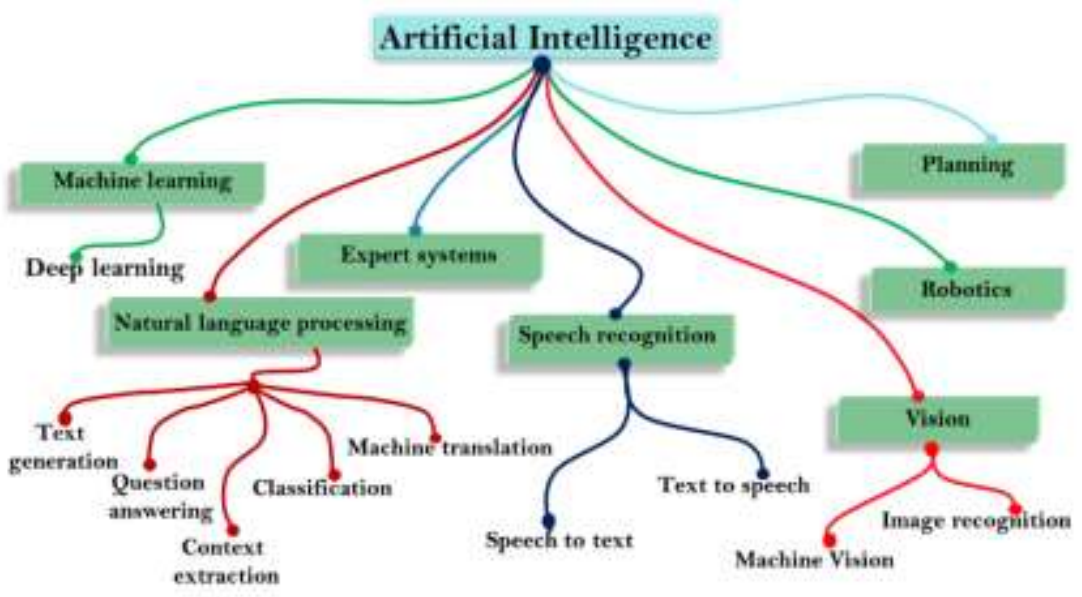

Figure 1. Artificial intelligence and its subfields (JavaTpoint, 2019)

$\mathrm{AI}$ is a broad and universal field which encompasses all kinds of intellectual tasks and has a huge variety of subfields from general, such as learning and perception, to those specific like playing chess, writing poetry, proving mathematical theorems, etc. (Russell, Norvig, 2016). The most important and most common among the branches of AI are: machine learning, deep learning, natural language processing, expert system, robotics, machine vision and speech recognition (JavaTpoint, 2019). Figure 1 shows different subfields of AI.

Machine learning can be found in most of the AI applications. It is a science of getting computers to learn like humans do, or simply, application of AI that makes systems able to learn and improve their learning over time by accessing data and information without being programmed (Expert system, 2019). There are three types of machine learning: supervised, unsupervised and deep learning. In supervised learning the system learns from training datasets, labelled as correct or incorrect, provided by user, while in unsupervised learning system uses statistical methods to group data with similar characteristics. There are many different types of machine learning algorithms and some of the most common are logistic regression, random forests, gradient boosting, support vector machines (SVMs), naive, and gradient boosting Bayes networks for supervised learning, and K-means clustering, principal component analysis and t-SNE for unsupervised learning (GFDRR, 2018).

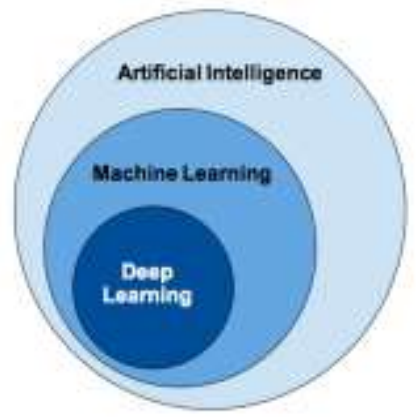

Figure 2. Artificial intelligence, machine learning and deep learning (Wasicek, 2018)

Deep learning (Figure 2) can be defined as a technique for implementing machine learning algorithms inspired by the structure and function of the human brain called artificial neural networks, which are composed of multiple processing layers to use multiple abstraction data (LeCun et al. 2015). There are many different types of artificial neural networks with different architectures designed for different purposes. Among them are convolutional neural networks, developed for image classification, that are usually applied for tasks involving spatial data (GFDRR, 2018).

In systems developed for spatial analysis, expert systems, natural language processing and computer vision can also be used. Expert system is a computer program that uses AI technologies, incorporating a knowledge base, to help judgement and decision making to an expert in some field (Rouse, 2016). Natural language processing enables machine to manipulate with natural language (speech and text), while computer vision enables the machines to "see", i.e. using cameras machines can capture and analyse visual information (Kumar, 2018). It is not easy to distinguish between AI subfields since they are interconnected and it is usually necessary to use more than one algorithm to accomplish the task of the system. For example, computer vision is often achieved through machine learning.

\section{AI IN GEOSPATIAL ANALYSIS IN DISASTER MANAGEMENT}

AI methods and algorithms have found their usage in all fields of expertise, and so did in the field of disaster management. Researchers are currently making great efforts to develop systems that can detect, and maybe one day predict ,earthquakes, which will be potentially enabled with the implementation of AI. Examples of using AI in disaster management can also be found in vulnerability and exposure assessments. Furthermore, AI is being used for processing information (such as satellite photos) and accelerating response when a disaster occurs. All of these applications require spatial data.

Geospatial analysis is an important part of disaster management that can be found in all of its phases, from mitigation to recovery. Due to the necessity for spatial data, there is a large number of projects that apply AI methods in spatial data analysis in disaster management. Table 1 presents the papers dealing with solving different tasks when dealing with catastrophes. In following chapters some of the case studies are presented more detailed. 


\begin{tabular}{|c|c|c|c|}
\hline Case study & Purpose of the analysis & AI algorithm & Input data \\
\hline Kia et al. 2012 & $\begin{array}{l}\text { Flood susceptibility } \\
\text { mapping }\end{array}$ & Artificial neural network & $\begin{array}{l}\text { Rainfall, slope, elevation, } \\
\text { flow accumulation, soil, } \\
\text { land use and geology data } \\
\text { layers from remote sensing }\end{array}$ \\
\hline Chen et al. 2017 & $\begin{array}{l}\text { Landslide susceptibility } \\
\text { mapping }\end{array}$ & $\begin{array}{l}\text { Rotation forest ensembles } \\
\text { (RFEs) and Naive Bayes } \\
\text { tree (NBT) classifiers }\end{array}$ & $\begin{array}{l}\text { Satellite images and field } \\
\text { survey }\end{array}$ \\
\hline Syifa et al. 2019 & $\begin{array}{l}\text { Post-earthquake damage } \\
\text { mapping }\end{array}$ & $\begin{array}{l}\text { Artificial neural network } \\
\text { (the back propagation } \\
\text { algorithm) and Support } \\
\text { vector machine (radial basis } \\
\text { function - RBF) }\end{array}$ & Satellite images \\
\hline Doshi et al. 2018 & $\begin{array}{l}\text { Priority assessment in } \\
\text { coordinating relief (flood } \\
\text { and fire) }\end{array}$ & $\begin{array}{c}\text { Convolutional Neural } \\
\text { Networks and semantic } \\
\text { segmentation models }\end{array}$ & Satellite images \\
\hline Vetrivel et al. 2018 & $\begin{array}{c}\text { Earthquake damage } \\
\text { detection }\end{array}$ & $\begin{array}{c}\text { Convolutional Neural } \\
\text { Networks }\end{array}$ & 3D point cloud \\
\hline Amit, Aoki, 2017 & $\begin{array}{l}\text { Landslide and flood disaster } \\
\text { detection }\end{array}$ & $\begin{array}{c}\text { Convolutional Neural } \\
\text { Networks }\end{array}$ & Satellite images \\
\hline Duarte et al. 2018 & $\begin{array}{c}\text { Classification of building } \\
\text { damages (earthquake) }\end{array}$ & $\begin{array}{c}\text { Convolutional Neural } \\
\text { Networks }\end{array}$ & Satellite and UAV images \\
\hline Ahmad et al. 2017 & $\begin{array}{c}\text { Disaster image retrieval } \\
\text { from social media and flood } \\
\text { detection in satellite images }\end{array}$ & $\begin{array}{c}\text { Convolutional Neural } \\
\text { Network, Support } \\
\text { Vector Machines (SVM), } \\
\text { Random forest } \\
\text { classification, Generative } \\
\text { adversarial network, }\end{array}$ & $\begin{array}{l}\text { Social media and satellite } \\
\text { images }\end{array}$ \\
\hline Ireland et al. 2015 & Flood area mapping & $\begin{array}{l}\text { Support vector machines } \\
\text { (SVM) and regularised } \\
\text { kernel Fisher's discriminant } \\
\text { analysis }\end{array}$ & Satellite images \\
\hline Cooner et al. 2016 & $\begin{array}{c}\text { Earthquake damage } \\
\text { detection }\end{array}$ & $\begin{array}{c}\text { Multilayer feedforward } \\
\text { neural networks, Radial } \\
\text { basis function neural } \\
\text { networks (RBFNN) and } \\
\text { Random Forests }\end{array}$ & Satellite images \\
\hline Lee et al. 2017 & Early fire detection & $\begin{array}{c}\text { Convolutional Neural } \\
\text { Networks }\end{array}$ & UAV images \\
\hline Nex et al. 2019 & $\begin{array}{l}\text { Near real-time damage } \\
\text { mapping }\end{array}$ & $\begin{array}{c}\text { Convolutional Neural } \\
\text { Networks }\end{array}$ & UAV images \\
\hline Petropoulos et al. 2010 & Burnt area mapping & Artificial neural network & Satellite images \\
\hline
\end{tabular}

Table 1. Case studies that implement AI in geospatial analysis in disaster management

\subsection{Disaster detection}

In their paper, Amit and Aoki (2017) proposed an automatic disaster detection system for landslide and flood detection, which is based on analysing satellite images with the application of convolutional neural network. Proposed disaster detection system is consisted of two phases (train phase and test phase) and uses Google Earth images as input data (Figure 3).

In the training phase system is learning all possible patterns of disasters through pre-disaster and post-disaster images with the same spatial information (same position). Training dataset for landslide detection was consisted of 18 sets (2200 patches/set), while the testing dataset included 17 sets (2200 patches/set). Flood detection training dataset involved 9 sets (2200 patches/set) and the testing set involved 13 sets (2200 patches/set). The patches were created by trimming $1072 \times 927$ pixels images into $32 \times 32$ pixels sized patches (Amit, Aoki, 2017).
Acording to test results, accuracy of the system of both flood and landslide detection is around $80 \%-90 \%$. The authors note that almost all of their images were taken during the sunny days, and if the images had combination of different color variation (snow, sunny day, rainy day) they would had been preprocessed (Amit, Aoki, 2017). 

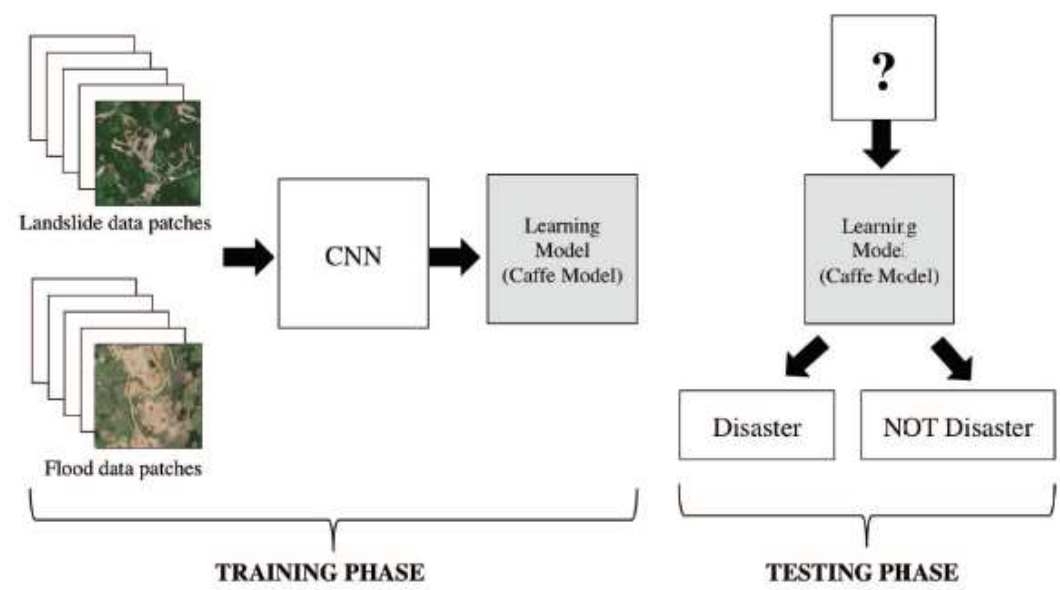

Figure 3. Two phases of the proposed system (Amit, Aoki, 2017)

\subsection{Coordinating relief efforts}

Doshi et al. (2018) proposed a framework for change detection on satellite images, using Convolutional Neural Networks $(\mathrm{CNN})$, which enables finding areas that are most affected by a disaster and helps coordinating relief efforts.

In order to locate areas with maximal damage, authors used models based on Fully-Convolutional Neural Networks that detect man-made features (roads and buildings) from satellite images and generate prediction masks. Man-made features are used in this research because they are changeless regarding season, lighting and noise difference in data obtained in different time series (Doshi et al. 2018).

Man-made features were extracted from before and after disaster images using a pre-trained semantic segmentation model. Prioritizing disaster response efforts is done by determining relative change in extracted features and computing

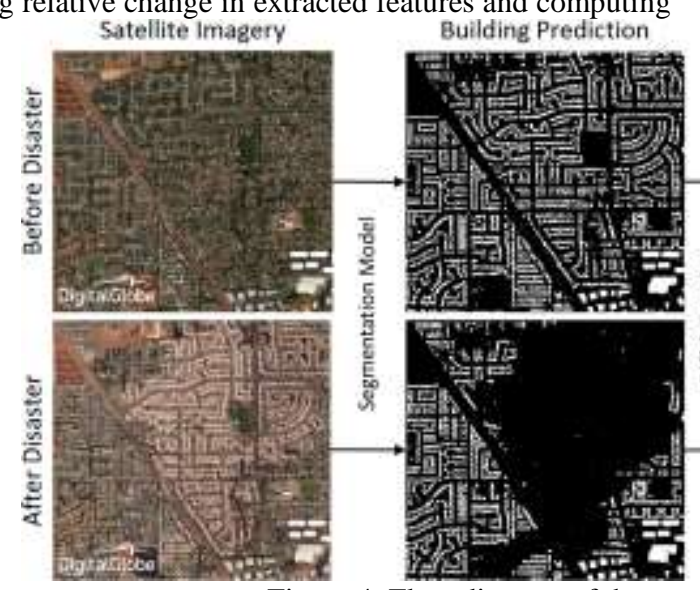

Figure 4. Flow diagram of the proposed approach (Doshi et al. 2018)
Disaster Impact Index (DII) - a metric to quantify impact of each area. Areas are divided into small grids, while DII is a normalized pixelwise change over the grid calculated according to number of pixels in the grid which contain detected feature in pre-disaster, but not in the post-disaster mask (Doshi et al. 2018).

A training dataset was consisted of two high-resolution satellite imagery sets - Spacenet and Deepglobe, and one additional dataset with lower resolution from Planet Labs for the road model training. Metodology was evaluated on Hurricane Harvey flood data and Santa Rosa fire data in three ways: comparing predicted pixelwise changes before and after disaster and pixelwise ground-truth labels, comparing DII with gridded ground-truth computed from pixelwise labels, comparing DII with labels for impact area (Doshi et al. 2018). Flow diagram of the proposed approach is shown in the Figure 4.

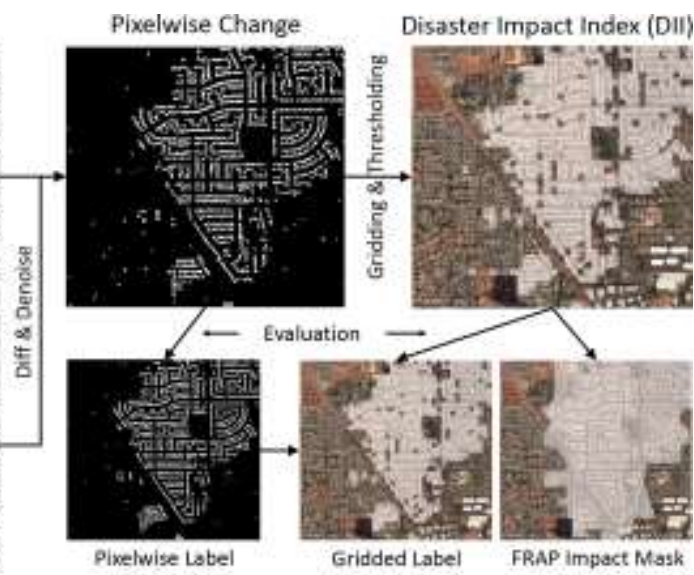

Results showed high correlation in detecting changes between both approaches (pixelwise and DII-based approach) and ground truth data. Authors concluded that DII can be used for prioritizing and coordination of disaster relief (Doshi et al. 2018).

\subsection{Urban damage detection}

In their study, Cooner et al. (2016) evaluated different algorithms for detecting earthquake damage caused by the 2010 Port-au-Prince, Haiti 7.0 moment magnitude (Mw) event, using remote sensing data. High resolution multispectral and panchromatic images, provided by DigitalGlobe Foundation were used in order to detect disaster damage: a pre-disaster panchromatic image, acquired by theWorldView-1 satellite and post-disaster multispectral and panchromatic images acquired by the Quickbird 2 satellite.

The study area was divided into two regions: training and validation region. To train the model authors manually digitized polygons over damaged structures and the total 134,327 damaged pixels were used. Number of undamaged pixels used 
in training the model was $1,214,623$. UNOSAT Haiti damage assessment was used for labelling over 900 buildings, as damaged or undamaged, in validation phase. Images were first preprocessed, extracting textural and structural information using different methods: entropy, dissimilarity, Laplacian or Gaussian and rectangular fit. After preprocessing training and validation datasets were transformed in different layers (prepanchromatic, pre-entropy, pre-dissimilarity, pre-LoG, prerectangular fit, post-panchromatic, post-entropy, postdissimilarity, post-LoG, post-rectangular fit, blue, green, red, and near infrared multispectral layers) whose values were normalized. Three machine learning algorithms were implemented: multilayer feedforward neural networks, radial basis function neural networks (RBFNN), and Random Forests (Cooner et al. 2016).

Two different datasets were used for validation process. On the first dataset, testing was performed using bulding-by-building accuracy assessment. The second dataset covered a larger area so the kernel density accuracy assessment was applied. Each of the three machine learning algorithms showed some positive results and authors stated that it is difficult to determine outright which of them is better, but they pointed out that feedforward neural network had the lowest error rate with the shortest runtime (Cooner et al. 2016).

\subsection{Post-disaster mapping}

In September 2018, an Mw 7.4 earthquake hit Donggala County in Inodnesia, which caused a tsunami and liquefaction in Palu City and Donggala, leading to loss of human lives and damaged buildings (Syifa et al. 2019). Syifa et al. (2019) presented a methodology for post-earthquake damage mapping using artificial neural network (the back propagation algorithm), support vector machine (radial basis function - RBF) classifiers and decorrelation method which were applied on the city of Palu (Indonesia). Post-earthquake map is the first step in risk assessment and planning evacuation and mitigation.

The data used in this study were pre-earthquake and postearthquake remote sensing images from Landsat- 8 and Sentinel2 , more precisely two images from Landsat-8, consisted of eight multispectral bands with a spatial resolution of $30 \mathrm{~m}$, and two Sentinel images with a spatial resolution of $10 \mathrm{~m}$ and three visible band (NIR, red and green). As it is shown in the Figure 5, ANN and SVM methods were used for map classification. Landsat images were divided into five classes: urban or affected area, vegetation, sea and rivers, bare land and clouds. Fifteen training samples (polygons) for each class were randomly created on a pixel-by-pixel basis. Sentinel images were categorized into six classes: urban or affected area, sea and river, vegetation, bare land, clouds, and shadows. The same training sampling methods as for the Landsat images were used (Syifa et al. 2019).

In order to produce post-earthquake maps, after the classification of the images, decorrelation method was applied. The method was adapted to differentiate pre-disaster and postdisaster images and to determine low similarity areas, which indicates changes, i.e. disaster damage (Syifa et al. 2019).

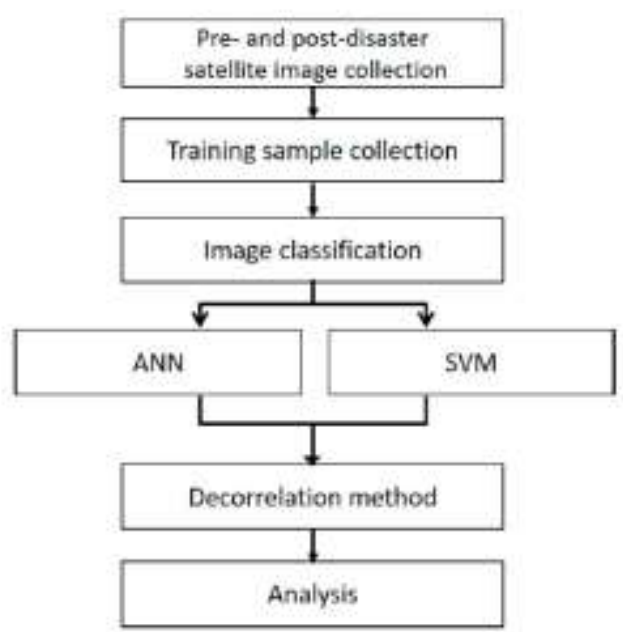

Figure 5. Flow chart of the method used to generate the damage maps (Syifa et al. 2019)

Four damage maps generated using proposed methodology were analysed and compared to field data. Analysis of conformity between the ANN and SVM results showed better results for Landsat imagery. In comparison with field data. Landsat images also showed superior results than did Sentinel images, in which the damaged areas were dominated by clouds (Syifa et al. 2019).

\section{CONCLUSION}

Due to the fact that about $80 \%$ of the data that are generated and used in different fields of life and science are geospatial in nature, practical application of AI is deeply tied with the geospatial world (Dasgupta 2017). Among the areas of application of AI is also geospatial analysis in disaster management.

As technology evolves and advances, there is a broader spectrum of sensors that provide spatial data. For quicker and enhanced integration and analysis of those data, and in order to get analysis results in near real-time and thus accelerate disaster response, AI algorithms are being increasingly used. In addition to fastening reactions when the catastrophe occurs, these algorithms can help in identifying risk areas and making better decisions in the future.

Based on the large number of scientific research projects, it can be said that this topic is really up-to-date and it will be interesting to see how these methodologies will evolve in the future.

\section{REFERENCES}

Ahmad, K., Pogorelov, K., Riegler, M., Conci, N., Halvorsen, P., 2017. CNN and GAN Based Satellite and Social Media Data Fusion for Disaster Detection. MediaEval'17, 13-15 September 2017, Dublin, Ireland.

Amit, S. N. K. B., Aoki, Y., 2017. Disaster detection from aerial imagery with convolutional neural network. In 2017 International Electronics Symposium on Knowledge Creation and Intelligent Computing (IES-KCIC), 239-245, IEEE.

Chen, W., Shirzadi, A., Shahabi, H., Ahmad, B. B., Zhang, S., Hong, H., Zhang, N., 2017. A novel hybrid artificial intelligence approach based on the rotation forest ensemble and 
naïve Bayes tree classifiers for a landslide susceptibility assessment in Langao County, China. Geomatics, Natural Hazards and Risk, 8(2), 1955-1977.

Cooner, A., Shao, Y., Campbell, J., 2016. Detection of urban damage using remote sensing and machine learning algorithms: Revisiting the 2010 Haiti earthquake. Remote Sensing, 8(10), 868-885.

Dasgupta, A., 2017. How AI is disrupting everything and where geospatial fits in? https://www.geospatialworld.net/article/ai-isdisrupting-where-geospatial-fit/. (5 June 2019)

Doshi, J., Basu, S., Pang, G., 2018. From satellite imagery to disaster insights. 32nd Conference on Neural Information Processing Systems (NIPS 2018), Montréal, Canada.

Duarte, D., Nex, F., Kerle, N., Vosselman, G., 2018. Satellite image classification of building damages using airborne and satellite image samples in a deep learning approach. ISPRS Annals of Photogrammetry, Remote Sensing \& Spatial Information Sciences, 4(2).

Expert system, 2019., What is machine learning? A definition, https://www.expertsystem.com/machine-learning-definition/ (20 July 2019).

GFDRR. 2018. Machine Learning for Disaster Risk Management. Washington, DC: GFDRR.

Ireland, G., Volpi, M., Petropoulos, G., 2015. Examining the capability of supervised machine learning classifiers in extracting flooded areas from Landsat TM imagery: A case study from a Mediterranean flood. Remote sensing, 7(3), 33723399 .

JavaTPoint, 2019. Artificial intelligence tutorial, https://www.javatpoint.com/artificial-intelligence-tutorial. (1 June 2019).

Joshi, N. 2019. 7 Types Of Artificial Intelligence. https://www.forbes.com/sites/cognitiveworld/2019/06/19/7types-of-artificial-intelligence/\#1649e90c233e. (20 June 2019).

Kia, M. B., Pirasteh, S., Pradhan, B., Mahmud, A. R., Sulaiman, W. N. A., Moradi, A., 2012. An artificial neural network model for flood simulation using GIS: Johor River Basin, Malaysia. Environmental Earth Sciences, 67(1), 251264.

Kumar, C. 2018. Artificial Intelligence: Definition, Types, Examples, Technologies. https://medium.com/@ chethankumargn/artificial-intelligencedefinition-types-examples-technologies-962ea75c7b9b. (1 June 2019).

LeCun, Y., Bengio, Y., Hinton, G., 2015. Deep learning. Nature, 521(7553), 436-445.

Lee, W., Kim, S., Lee, Y. T., Lee, H. W., Choi, M., 2017. Deep neural networks for wild fire detection with unmanned aerial vehicle. In 2017 IEEE international conference on consumer electronics (ICCE), 252-253, IEEE.

McCarthy, J., Minsky, M., Rochester, N., Shannon, C., 1955. A proposal for the dartmouth summer research project on artificial intelligence.

http://www-

formal.stanford.edu/jmc/history/dartmouth/dartmouth.htm. (15 June 2019).

McCharty, J., 2007. What is artificial intelligence? Technical report, Computer Science Department, Stanford University.

Mellit, A., Kalogirou, S. A., 2008. Artificial intelligence techniques for photovoltaic applications: A review. Progress in energy and combustion science, 34(5), 574-632.

Nex, F., Duarte, D., Steenbeek, A., Kerle, N., 2019. Towards Real-Time Building Damage Mapping with Low-Cost UAV Solutions. Remote sensing, 11(3), 287-301.

Petropoulos, G. P., Vadrevu, K. P., Xanthopoulos, G., Karantounias, G., Scholze, M., 2010. A comparison of spectral angle mapper and artificial neural network classifiers combined with Landsat TM imagery analysis for obtaining burnt area mapping. Sensors, 10(3), 1967-1985.

Ray, S., 2018. History of AI. https://towardsdatascience.com/history-of-ai-484a86fc16ef. (15 June 2019).

Rouse M., 2016., Expert system, https://searchenterpriseai.techtarget.com/definition/expertsystem. (1 June 2019).

Russell, S. J., Norvig, P., 2016. Artificial intelligence: a modern approach. Malaysia; Pearson Education Limited.

Syifa, M., Kadavi, P. R., Lee, C. W., 2019. An Artificial Intelligence Application for Post-Earthquake Damage Mapping in Palu, Central Sulawesi, Indonesia. Sensors, 19(3), 542-560.

UNITAR/UNOSAT, EC JRC, and World Bank. Haiti Earthquake 2010: Remote Sensing Damage Assessment. http://www.unitar.org/unosat/haiti-earthquake-2010-remotesensing-based-buildingdamage-assessment-data. (5 June 2019).

Vetrivel, A., Gerke, M., Kerle, N., Nex, F., Vosselman, G., 2018. Disaster damage detection through synergistic use of deep learning and 3D point cloud features derived from very high resolution oblique aerial images, and multiple-kernel-learning. ISPRS journal of photogrammetry and remote sensing, 140, 4559.

Wasicek, A. 2018. Artificial Intelligence vs. Machine Learning vs. Deep Learning: What's the Difference? https://www.sumologic.com/blog/machine-learning-deeplearning/. (1 June 2019) 